Cite this: Phys. Chem. Chem. Phys., 2011, 13, 8448-8456

\title{
Crossed beam scattering experiments with optimized energy resolution
}

\author{
Ludwig Scharfenberg, Sebastiaan Y. T. van de Meerakker* and Gerard Meijer
}

DOI: $10.1039 / \mathrm{c0cp} 02405 \mathrm{~h}$

Crossed molecular beam scattering experiments in which the energy of the collision is varied can reveal valuable insight into the collision dynamics. The energy resolution that can be obtained depends mainly on the velocity and angular spreads of the molecular beams; often, these are too broad to resolve narrow features in the cross sections like scattering resonances. The collision energy resolution can be greatly improved by making appropriate choices for the beam velocities and the beam intersection angle. This method works particularly well for situations in which one of the beams has a narrow velocity spread, and we here discuss the implications of this method for crossed beam scattering experiments with Stark-decelerated beams.

\section{Introduction}

The crossed molecular beam technique is one of the most widely used experimental approaches to study collisions between individual atoms and molecules, and has been seminal to our present understanding of molecular dynamics at a microscopic level. ${ }^{1}$ Since its introduction in the 1950's, the technique has witnessed a remarkable and continuous development. Its present level of advancement allows for accurate control over the collision partners prior to the collision event, and for sophisticated detection of the collision products. $^{2-4}$

One of the most important parameters in a collision experiment is the collision energy of the colliding particles. The collision energy can be tuned by controlling the velocity of the particles prior to the collision, or by changing the angle between the intersecting beams. For the latter approach, ingenious crossed molecular beam machines have been engineered to continuously vary the collision energy. ${ }^{5}$ These methods have been used to measure, for instance, the threshold behavior of rotational energy transfer, ${ }^{6,7}$ or to tune the collision energy over the reaction barrier for reactive scattering. 8,9

Recently, new molecular beam techniques have become available that allow for detailed control over the velocity of molecules in a beam. This control is obtained by exploiting the interaction of molecules with electric or magnetic fields in a so-called Stark decelerator or Zeeman decelerator, respectively. ${ }^{10}$ The tunability of the velocity allows for scanning of the collision energy in a fixed experimental geometry. State-to-state inelastic scattering between Stark-decelerated $\mathrm{OH}$ radicals and conventional beams of $\mathrm{Xe}, \mathrm{Ar}$, and $\mathrm{He}$ atoms, as well as $\mathrm{D}_{2}$ molecules, ${ }^{1-13}$ has been studied. These beam deceleration methods hold great promise for future scattering experiments

Fritz-Haber-Institut der Max-Planck-Gesellschaft, Faradayweg 4-6, 14195 Berlin, Germany.E-mail: basvdm@fhi-berlin.mpg.de and offer the possibility to extend the available collision energy range to energies below one wavenumber. ${ }^{14}$

Essential in these experiments is the resolution with which the collision energy can be varied. High energy resolutions are particularly important at those collision energies where a detailed structure in the energy dependence of the cross sections is expected. At low collision energies, shape or orbiting resonances can occur that are caused by rotational states of the collision complex that are trapped behind the centrifugal or reaction barrier. ${ }^{15,16}$ At collision energies near the energies of excited states of one of the reagents, also Feshbach resonances can occur. ${ }^{17}$ The experimental mapping of these resonances would probe the potential energy surfaces that govern the interactions with unprecedented detail. ${ }^{18}$

The energy resolution that can be obtained experimentally depends on the velocity and angular spreads of the molecular beam pulses. Typical molecular beam spreads are too large to reveal narrow features like scattering resonances that often require energy resolutions of about one wavenumber. So far, only in exceptional cases have resonances been observed, mostly for kinematically favorable systems in which a collision partner with low mass has been used. ${ }^{19-22}$ Recently, crossed beam experiments employing a tunable beam crossing angle have been reported in which the resolution was sufficient to resolve the contribution of individual partial waves to the scattering. ${ }^{23,24}$

Compared to conventional molecular beams, Stark-decelerated molecular beams offer superior velocity spreads that typically range between 1 and $20 \mathrm{~m} \mathrm{~s}^{-1} .^{25}$ This narrow velocity spread can be exploited in crossed beam scattering experiments to yield a high energy resolution. Indeed, energy resolutions of $\geq 13 \mathrm{~cm}^{-1}$ have already been achieved for the $\mathrm{OH}-\mathrm{Xe}$ and $\mathrm{OH}-\mathrm{Ar}$ systems, which is particularly good in view of the relatively large reduced mass of these systems. This energy resolution was sufficient to accurately measure the threshold behavior of the rotational inelastic cross sections, ${ }^{11}$ and to resolve broader features in the collision energy dependence of 
the cross sections. ${ }^{12}$ The sharp resonances that are predicted by ab initio calculations remained elusive, however.

To further improve the energy resolution in these experiments, the velocity spread of the collision partner needs to be reduced. This can be achieved by using a second Stark decelerator to obtain control over a molecular collision partner, or by using mechanical velocity selectors to reduce the velocity spread of the atomic collision partner. However, both approaches would greatly reduce the number density in the colliding beam.

Here we describe a simple yet effective method to improve the collision energy resolution that does not rely on velocity selection of the target beam. We show that for beam crossing angles smaller than $90^{\circ}$, kinematically favorable situations can occur in which the velocity spread of the target beam does not contribute to the collision energy resolution. This enables high collision energy resolutions without sacrificing the number density of the target beam that is available to the scattering.

This method has been exploited before to improve the resolution in scattering experiments. To the best of our knowledge, it was described for the first time in a book chapter by Pauly and Toennies ${ }^{26}$ in 1968 and it was part of the dissertation $^{27}$ of R. Feltgen (a student of Pauly) in 1970. The method was used in an experiment by Scoles and coworkers, in which orbiting resonances were observed in the integral elastic scattering cross sections for the scattering of velocity selected $\mathrm{H}$ atoms by $\mathrm{Hg}$ atoms. ${ }^{19,20} \mathrm{~A}$ beam intersection angle of $73^{\circ}$ was used in order to improve the velocity resolution. A similar investigation was performed by Toennies and coworkers, who used a beam intersection angle of $46^{\circ}$ to resolve orbiting resonances in the scattering of $\mathrm{H}$ atoms by various rare gas atoms. ${ }^{21}$ It is noted that a smart use of the beam kinematics has also been exploited to optimize the post-collision velocity spread of the scattered molecules. ${ }^{28,29}$

The method is particularly advantageous if one of the beams has a narrow velocity spread. For collisions between Stark-decelerated beams and conventional beams of rare gas atoms, for instance, a very high energy resolution can be obtained that may well be sufficient to experimentally resolve scattering resonances, even for systems with a relatively large reduced mass.

This paper is organized as follows. In Section II the method is explained in more detail, and the beam properties that are used throughout this paper are introduced. In Section III we describe different experimental approaches that can be followed to vary the collision energy, and their implications for the collision energy resolution are analyzed. The description will be held rather generally, although we will emphasize on the experimental arrangement of one Stark-decelerated beam colliding with a conventional molecular beam. In Section IV we illustrate the potential of the method using a recent crossed beam experiment as an example. In this experiment, a Stark decelerated beam of $\mathrm{OH}$ radicals was scattered with a beam of $\mathrm{He}$ atoms at a $90^{\circ}$ crossing angle, and we show that the future implementation of the method may well lead to the experimental observation of scattering resonances for this system. In Section V we will draw conclusions, again with an emphasis on the advantages this method can have for crossed beam collision experiments in which Stark-decelerated beams are employed.

\section{Collision kinematics}

Consider two colliding particles with mass $m_{1}$ and $m_{2}$ and with laboratory velocity vectors $\mathbf{v}_{1}$ and $\mathbf{v}_{2}$, respectively. This situation is schematically represented in Fig. 1. The collision energy $E$ of the system, calculated in a frame of reference that is moving with the velocity of the center-of-mass of the two particles, is given by:

$$
E=\frac{\mu}{2}\left|\mathbf{v}_{1}-\mathbf{v}_{2}\right|^{2}=\frac{\mu}{2}\left(v_{1}^{2}+v_{2}^{2}-2 v_{1} v_{2} \cos \phi\right),
$$

where $v_{1}$ and $v_{2}$ are the magnitudes of the laboratory velocity vectors, $\phi$ is the enclosed angle between both velocity vectors, and $\mu=m_{1} m_{2} /\left(m_{1}+m_{2}\right)$ is the reduced mass of the system. This energy $E$ is the total energy that is available for inelastic processes. Small changes in $v_{1}, v_{2}$ or $\phi$ will cause an approximate change of $E$ that is given by its differential:

$\mathrm{d} E=\mu\left(\left[v_{1}-v_{2} \cos \phi\right] \mathrm{d} v_{1}+\left[v_{2}-v_{1} \cos \phi\right] \mathrm{d} v_{2}+v_{1} v_{2} \sin (\phi) \mathrm{d} \phi\right)$.

The geometric meaning of the partial derivatives is brought out more clearly if expressed directly by the velocity vectors:

$\mathrm{d} E=\mu\left(\left[v_{1}-\hat{\mathbf{v}}_{1} \cdot \mathbf{v}_{2}\right] \mathrm{d} v_{1}+\left[v_{2}-\hat{\mathbf{v}}_{2} \cdot \mathbf{v}_{1}\right] \mathrm{d} v_{2}+\left|\mathbf{v}_{1} \times \mathbf{v}_{2}\right| \mathrm{d} \phi\right)$

with the vectors of unit length $\hat{\mathbf{v}}_{1}$ and $\hat{\mathbf{v}}_{2}$.

Two important special cases can occur. If the beams are parallel on average $\left(\phi=0^{\circ}\right.$ or $\left.180^{\circ}\right)$, the influence of the angular spread of the beams becomes negligible. If the relative velocity vector $g$ is, on average, perpendicular to $\mathbf{v}_{1}$ or $\mathbf{v}_{2}, E$ is almost unaffected by small changes in $v_{1}$ or $v_{2}$, respectively. In this case the influence of the velocity spread of one of the beams becomes negligible. The collision energy resolution thus strongly depends on the geometry of the Newton diagram that describes the scattering process. For a suitable choice of the geometry, this can be exploited to optimize the collision energy resolution in the experiment. This is the main idea behind the method.

To make the discussion quantitative, an estimate of the collision energy distribution is required. This distribution is determined by the distributions of the vectors $\mathbf{v}_{1}$ and $\mathbf{v}_{2}$ and hence by six independent variables. This number can be reduced by changing to a more suitable coordinate system

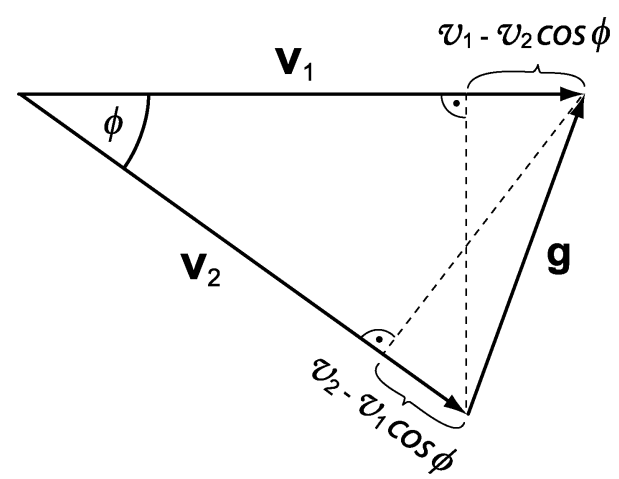

Fig. 1 Laboratory velocity vectors $\mathbf{v}_{1}$ and $\mathbf{v}_{2}$ of two colliding particles. The relative velocity vector of the two particles is given by the vector $\mathbf{g}=\mathbf{v}_{1}-\mathbf{v}_{2}$. 
Table 1 Reduced mass $\mu=m_{1} m_{2} /\left(m_{1}+m_{2}\right)$ (in atomic units) for a selection of collision systems

\begin{tabular}{|c|c|c|c|c|}
\hline \multirow{2}{*}{$m_{2} / m_{1}$} & & \multirow{2}{*}{$\frac{{ }^{7} \mathrm{LiH}}{8}$} & \multirow{2}{*}{$\frac{\mathrm{OH} / \mathrm{NH}_{3}}{17}$} & \multirow{2}{*}{$\frac{\mathrm{CO}}{28}$} \\
\hline & & & & \\
\hline 1 & $\mathrm{H}$ & 0.88 & 0.94 & 0.97 \\
\hline 2 & $\mathrm{D} / \mathrm{H}_{2}$ & 1.60 & 1.79 & 1.87 \\
\hline 3 & ${ }^{3} \mathrm{He} / \mathrm{HD}$ & 2.18 & 2.55 & 2.71 \\
\hline 4 & ${ }^{4} \mathrm{He} / \mathrm{D}_{2}$ & 2.67 & 3.24 & 3.50 \\
\hline 8 & ${ }^{7} \mathrm{LiH}$ & 4.00 & 5.44 & 6.22 \\
\hline 17 & $\mathrm{OH} / \mathrm{NH}_{3}$ & 5.44 & 8.50 & 10.58 \\
\hline 20 & $\mathrm{Ne} / \mathrm{ND}_{3}$ & 5.71 & 9.19 & 11.67 \\
\hline 28 & $\mathrm{CO}$ & 6.22 & 10.57 & 14.00 \\
\hline 40 & $\mathrm{Ar}$ & 6.67 & 11.93 & 16.47 \\
\hline 83.8 & $\mathrm{Kr}$ & 7.30 & 14.13 & 20.99 \\
\hline 131.3 & $\mathrm{Xe}$ & 7.54 & 15.05 & 23.08 \\
\hline
\end{tabular}

and by making appropriate approximations. If the vectors $\mathbf{v}_{1}$ and $\mathbf{v}_{2}$ are written as functions of spherical coordinates the collision energy becomes:

$$
E\left(\mathbf{v}_{1}, \mathbf{v}_{2}\right)=E\left(\mathbf{v}_{1}\left(v_{1}, \varphi_{1}, \theta_{1}\right), \mathbf{v}_{2}\left(v_{2}, \varphi_{2}, \theta_{2}\right)\right)
$$

where $\theta_{1(2)}$ denotes the polar angle, i.e. the angle subtended by $\mathbf{v}_{1(2)}$ and the $z$-axis and $\varphi_{1(2)}$ denotes the azimuthal angle, i.e. the angle subtended by the orthogonal projection of $\mathbf{v}_{1(2)}$ onto the $x y$-plane and the $x$-axis. If the averaged velocity vectors lie exactly within the $x y$-plane, the first order change of $E$ with $\theta_{1(2)}$ vanishes ${ }^{35}$ so that we need to consider the projection of the velocity vectors onto the $x y$-plane only. For the experiment, this means that it is sufficient to collimate the beams by slits (rather than pinholes) that are oriented perpendicular to the $x y$-plane. We can now identify $\phi$ in eqn (1) with $\phi=\varphi_{1}-\varphi_{2}$, and we only have to optimize the collision energy resolution with respect to the three scalar variables $v_{1}, v_{2}$, and $\phi$.

In the experiment, $v_{1}, v_{2}$ and $\phi$ are distributed around their mean values; let the variance of these variables be denoted by $\sigma .{ }^{36}$ Because $\varphi_{1}$ and $\varphi_{2}$ are independent, the variance $\sigma_{\phi}$ of the distribution for $\phi$ is given by $\sigma_{\phi}^{2}=\sigma_{\varphi_{1}}^{2}+\sigma_{\varphi_{2}}^{2}$. Hence the differential (2) can be used to estimate the width of the energy distribution, and the variance of the collision energy, $\sigma(E)$, is given to first order by:

$$
\begin{aligned}
\sigma^{2}(E)= & \mu^{2}\left(\left[v_{1}-v_{2} \cos \phi\right]^{2} \sigma_{v_{1}}^{2}+\left[v_{2}-v_{1} \cos \phi\right]^{2} \sigma_{v_{2}}^{2}\right. \\
& \left.+\left[v_{1} v_{2} \sin \phi\right]^{2} \sigma_{\phi}^{2}\right)
\end{aligned}
$$

in which $v_{1}, v_{2}$ and $\phi$ now stand for the respective mean values.

Because $E$ as well as $\sigma(E)$ is linear in $\mu$, it suffices to consider $\sigma(E / \mu)$. For convenience, the value of $\mu$ is listed in Table 1 for a few selected collision systems. The molecules that are listed in the top row are typical molecules that are suitable candidates for Stark deceleration.

\section{Overview and applications}

If one intends to conduct an experiment at a given mean energy $E$ with the highest possible resolution, one has to optimize five parameters: $\Delta v_{1}, \Delta v_{2}, \Delta \phi$ and the mean values of two of the three variables $v_{1}, v_{2}, \phi$ - the third is always determined through eqn (1). In the following sections, we will analyze how the resolution depends on the experimental parameters, using three different experimental approaches. In Section IIIA we discuss the situation in which the beam speeds are held constant, and the collision energy is tuned by variation of the beam intersection angle $\phi$ alone. In Section IIIB, we describe the situation for a fixed beam intersection angle and target beam speed; the collision energy is tuned by variation of $v_{1}$. Finally, in Section IIIC we discuss the most general case in which $v_{1}, v_{2}$, and $\phi$ are allowed to vary to optimize the energy resolution.

The parameters that are used in the examples are chosen to represent the collision energy resolution as realistic as possible and that may be expected in an experiment. The molecular beam velocity spreads are assumed to be $10 \%$ of the mean speed of the beam. In those cases where the velocity of the primary beam $\left(v_{1}\right)$ is varied, we assume that the beam is produced with a Stark decelerator. For a Starkdecelerated beam, the absolute velocity spread in the forward direction is (almost) constant and does not depend on the mean velocity; we will assume here a constant velocity spread of $10 \mathrm{~m} \mathrm{~s}^{-1}$ for all cases. The angular spread of a Stark-decelerated beam is generally smaller (typically $1^{\circ}$, or about $20 \mathrm{mrad}$ ) than the angular spread of a conventional molecular beam. To simplify the analysis, we assume a constant angular spread in our examples, but one should keep in mind that it actually depends on the forward velocity if a decelerator is used. Angular spreads are assumed to be 0 , 20,40 or $80 \mathrm{mrad}$.

In our analysis, we assume Gaussian distributions for all variables. In this case the distribution for $E$, as approximated by the differential, becomes a well defined Gaussian with $\sigma(E)$ given by eqn (5). If we denote the full width at half maximum of the distribution of quantity $x$ by $\Delta(x) \equiv \Delta_{x}$, we have $\Delta(x)=$ $2.35 \sigma(x)$ and

$$
\begin{aligned}
\Delta(E / \mu)= & \left(\left[v_{1}-v_{2} \cos \phi\right]^{2} \Delta_{v_{1}}^{2}+\left[v_{2}-v_{1} \cos \phi\right]^{2} \Delta_{v_{2}}^{2}\right. \\
& \left.+\left[v_{1} v_{2} \sin \phi\right]^{2} \Delta_{\phi}^{2}\right)^{1 / 2} .
\end{aligned}
$$

This expression is used for all calculations that are presented below.

\section{A $\quad v_{1}$ and $v_{2}$ constant, $\phi$ variable}

In this case, both beam speeds are assumed to be constant, and the beam intersection angle alone is used to change the energy. For the kinematic parameters we use $v_{1}=v_{2}=500 \mathrm{~m} \mathrm{~s}^{-1}$, and $\Delta v_{1}=\Delta v_{2}=50 \mathrm{~m} \mathrm{~s}^{-1}$. The resulting curves for the energy resolution $\Delta(E / \mu)$ as a function of $E / \mu$ are shown in Fig. 2. Two curves are shown that correspond to an angular spread of $\Delta \phi=0$ (red dashed curve) and $\Delta \phi=40 \mathrm{mrad}$ (red solid curve). The angle $\phi$ that is needed to obtain a specific $E / \mu$ is given by the black curve with respect to the right axis.

If small crossing angles can be realized, fairly low collision energies are accessible for systems with a small reduced mass. For example, the system $\mathrm{OH} /{ }^{4} \mathrm{He}$ has $\mu=3.2 \mathrm{u}$, so that at $30^{\circ}$ a collision energy of $\sim 9 \mathrm{~cm}^{-1}$ is obtained with a resolution of $\sim 1.9 \mathrm{~cm}^{-1}$.

The energy resolution $\Delta(E / \mu)$ depends approximately linearly on the energy $E / \mu$, resulting in a constant relative energy resolution $\Delta E / E$. This linear behavior is a consequence of 


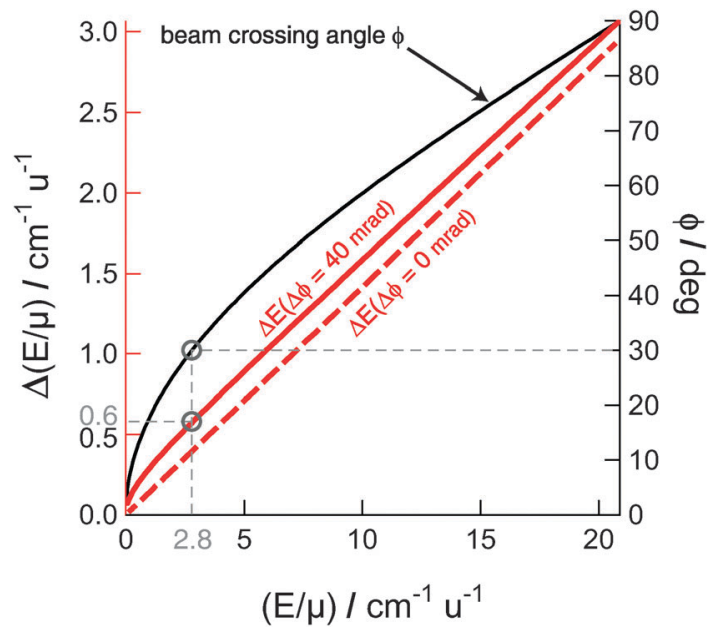

Fig. 2 The dependence of the full width at half maximum $\Delta(E / \mu)$ on $E / \mu$ pertaining to the situation in which both beam velocities are constant and the collision energy is tuned by variation of $\phi$ (see Section IIIA). Beam parameters: $v_{1}=v_{2}=500 \mathrm{~m} \mathrm{~s}^{-1}, \Delta_{v_{1}}=\Delta_{v_{2}}=$ $50 \mathrm{~m} \mathrm{~s}^{-1}$ and $\Delta_{\phi}=40 \mathrm{mrad}\left(2.3^{\circ}\right)$ (solid red curve), $\Delta_{\phi}=0 \mathrm{mrad}$ (dashed red curve). The corresponding beam intersection angle is shown as the black curve with respect to the axis on the right side.

the choice of equal velocities $v_{1}=v_{2}=v$. With the help of eqn (6) and (1), the relative energy resolution $\Delta E / E$ for this special case is given by:

$$
\frac{\Delta E}{E}=\left(\frac{\Delta_{v_{1}}^{2}+\Delta_{v_{2}}^{2}}{v^{2}}+\frac{1+\cos \phi}{1-\cos \phi} \Delta_{\phi}^{2}\right)^{1 / 2},
$$

which is nearly independent of $\phi$ for small values of $\Delta \phi$.

It is noted that the low collision energies and high energy resolutions that can be obtained for small beam intersection angles and systems with low reduced mass have been exploited recently in an experiment by Costes and coworkers, who have thereby been able to observe oscillations in the integral cross sections for the reactive scattering of $\mathrm{S}\left({ }^{1} \mathrm{D}_{2}\right)$ atoms with $\mathrm{H}_{2}$ molecules. ${ }^{24}$

\section{B $\quad v_{2}$ and $\phi$ constant, $v_{1}$ variable}

In this case, the experimental geometry and the target beam velocity are fixed and the collision energy is tuned by varying the velocity $v_{1}$. This situation pertains, for instance, to a collision experiment in which a Stark-decelerated beam is collided with a conventional molecular beam at a fixed beam intersection angle. Hence we assume in our analysis for beam 2 the parameters $v_{2}=500 \mathrm{~m} \mathrm{~s}^{-1}$ and $\Delta_{v_{2}}=50 \mathrm{~m} \mathrm{~s}^{-1}$; for beam 1 we assume a velocity spread of $\Delta_{v_{1}}=10 \mathrm{~m} \mathrm{~s}^{-1}$ for all velocities. Further, we assume an angular spread $\Delta_{\phi}=40$ $\operatorname{mrad}\left(2.3^{\circ}\right)$.

In Fig. 3 the resulting values for $\Delta(E / \mu)$ are shown for two different beam intersection angles. The red solid and red dashed curves (with respect to the axis on the left) show the expected collision energy resolution as a function of the collision energy for $\phi=45^{\circ}$ and $\phi=90^{\circ}$, respectively. The corresponding primary beam velocities $v_{1}$ that are required to obtain this collision energy are shown as green curves with respect to the axis on the right.

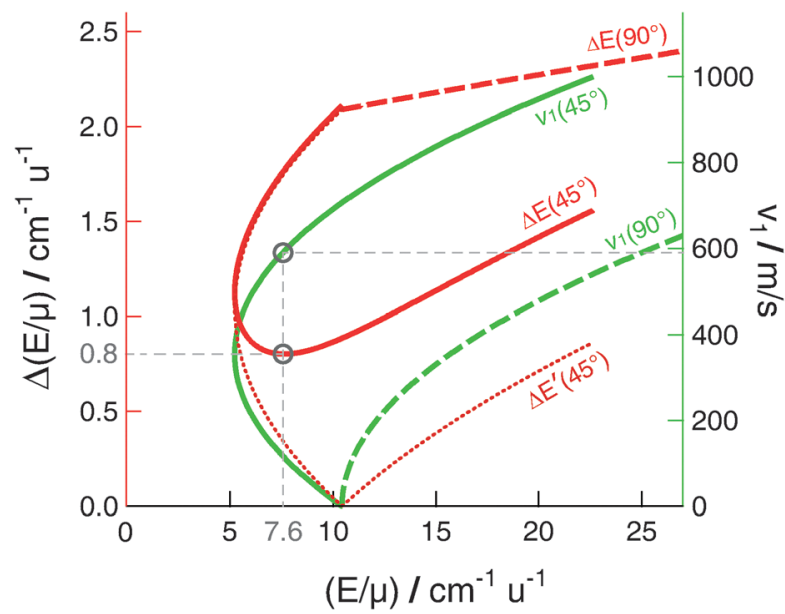

Fig. 3 The dependence of $\Delta(E / \mu)$ on $E / \mu$ for the situation in which the beam intersection angle and the target beam velocity $v_{2}$ are constant, while the collision energy is tuned by variation of $v_{1}$ (see Section IIIB). Beam intersection angles of $\phi=45^{\circ}$ (red solid curve) or $\phi=90^{\circ}$ (red dashed curve) are assumed. Beam parameters: $v_{2}=$ $500 \mathrm{~m} \mathrm{~s}^{-1}, \Delta_{v_{2}}=50 \mathrm{~m} \mathrm{~s}^{-1}, \Delta_{v_{1}}=10 \mathrm{~m} \mathrm{~s}^{-1}$, and $\Delta_{\phi}=40 \mathrm{mrad}\left(2.3^{\circ}\right)$. The corresponding primary beam velocities $v_{1}$ are shown as the green curves with respect to the axis on the right. Note that at low collision energies for $45^{\circ}$ there are two possible values for $v_{1}$ at a given energy with differing values for the resolution. The red dotted curve labeled $\Delta E^{\prime}\left(45^{\circ}\right)$ pertains to the hypothetical case in which $\Delta v_{1}=0 \mathrm{~m} \mathrm{~s}^{-1}$, $\Delta \phi=0$ mrad.

From Fig. 3 it is evident that beam crossing angles of $\phi=$ $45^{\circ}$ result in lower collision energies, and, perhaps more important, better energy resolutions. At low collision energies, there are actually two values for $v_{1}$ that result in the same collision energy. The energy resolution, however, is much different for both situations. The energy resolution shows a minimum that occurs for the chosen beam parameters at $E / \mu=7.6 \mathrm{~cm}^{-1} \mathrm{u}^{-1}$ and $v_{1}=600 \mathrm{~m} \mathrm{~s}^{-1}$.

From the analysis given in Section II, one would expect that the best collision energy resolution is obtained when the relative velocity vector $g$ is perpendicular to $\mathbf{v}_{2}$; this condition is fulfilled for $E / \mu=10.4 \mathrm{~cm}^{-1} \mathrm{u}^{-1}$ and $v_{1}=707 \mathrm{~m} \mathrm{~s}^{-1}$. The position of the minimum that is found in Fig. 3 deviates slightly from these values due to the nonzero angular spread $\Delta_{\phi}$ and the nonzero velocity spread of beam 1. This is illustrated by the red dotted curve in Fig. 3, labeled $\Delta E^{\prime}\left(45^{\circ}\right)$, that shows the energy resolution that would be obtained for $\Delta_{v_{1}}=\Delta_{\phi}=0$. In this hypothetical situation, the best collision energy resolution that can be obtained is indeed found for $\mathbf{g} \perp \mathbf{v}_{2}$, and becomes independent of the velocity spread of beam 2 . To first order, the collision energy spread vanishes in this case.

\section{Variation of $v_{1}, v_{2}$, and $\phi$ for a fixed energy}

In this case the mean collision energy is specified while $v_{1}, v_{2}$, and $\phi$ are allowed to vary. For a given choice of $E, v_{1}$ and $\phi$, there are in general two possible values for $v_{2}$ which yield this energy $E$. In calculations it is therefore advantageous to vary $v_{1}$ and $v_{2}$ and to let $\phi$ be uniquely determined by eqn (1). To search for a minimum in $\Delta E$ then has the following geometrical significance: the vectors $\mathbf{v}_{1}, \mathbf{v}_{2}$ and the relative velocity $\mathbf{g}$ define 


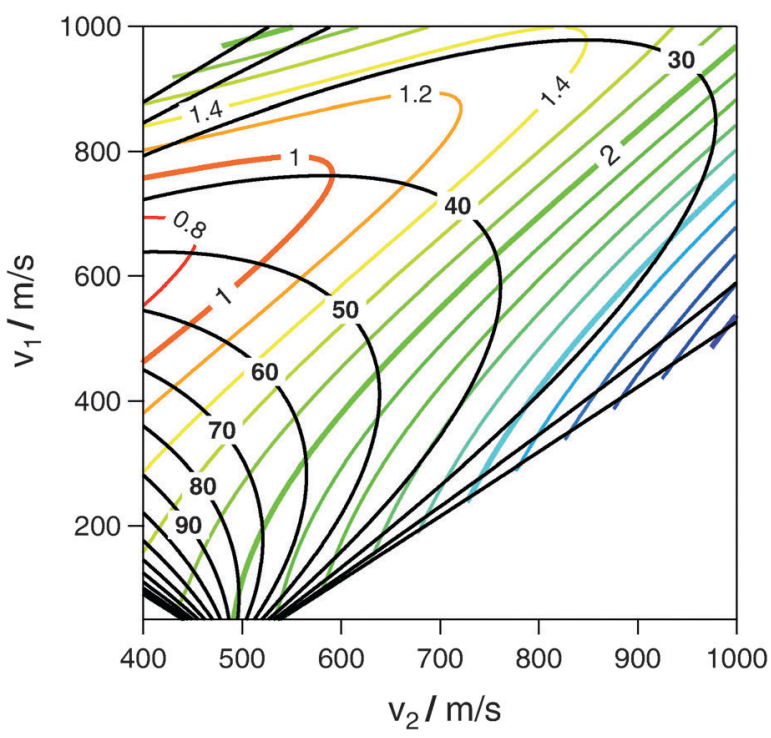

Fig. 4 Contour plot of $\Delta(E / \mu)$ for a fixed $E / \mu$ of $10 \mathrm{~cm}^{-1} \mathrm{u}^{-1}$ pertaining to the situation where $v_{1}, v_{2}$, and $\phi$ are varied (see Section IIIC). Beam parameters: $\Delta_{v_{1}}=10 \mathrm{~m} \mathrm{~s}^{-1}, \Delta_{v_{2}}=0.10 \times v_{2}, \Delta_{\phi}=40 \mathrm{mrad}$. The contour lines for the beam intersection angles $\phi$ are shown as black curves.

a triangle, $\mathbf{g}$ is held fixed and the vertex opposite to $\mathbf{g}$ is allowed to move over all points within the plane (excluding some areas which may not be accessible in the experiment).

Again, we calculate the expected energy resolution for an experiment in which a Stark-decelerated beam collides with a conventional molecular beam; i.e., we take the beam parameters $\Delta_{v_{1}}=10 \mathrm{~m} \mathrm{~s}^{-1}, \Delta_{v_{2}}=0.10 \times v_{2}$ and $\Delta_{\phi}=40 \mathrm{mrad}$. The collision energy resolution $\Delta(E / \mu)$ is calculated on a sufficiently fine grid of values for $v_{1}$ and $v_{2}$, where $v_{1}=100-1000 \mathrm{~m} \mathrm{~s}^{-1}$ and $v_{2}=400-1000 \mathrm{~m} \mathrm{~s}^{-1}$. The subsidiary condition of constant energy is introduced by letting $\phi$ be determined by eqn (1). The surface $\Delta(E / \mu)\left(v_{1}, v_{2}\right)$ for the fixed collision energy $E / \mu=10 \mathrm{~cm}^{-1} \mathrm{u}^{-1}$ is shown in Fig. 4.

The optimal resolution with $\Delta(E / \mu)=0.73 \mathrm{~cm}^{-1} \mathrm{u}^{-1}$ is obtained for $v_{1}=627 \mathrm{~m} \mathrm{~s}^{-1}, v_{2}=400 \mathrm{~m} \mathrm{~s}^{-1}$ and $\phi=51^{\circ}$; note that there is no local minimum, only a global one.

\section{Applications}

In a crossed beam collision experiment, one would like to tune the collision energy with the highest possible resolution for each value of the collision energy. As described in Section IIIC, one would have to optimize the values for $v_{1}, v_{2}$, and $\phi$ to accomplish this. This is possible in theory, it is however not practical in an experiment. In this section we discuss to which extent satisfactory results can also be obtained by a variation of two parameters only.

First, let us assume that the apparatus allows for a continuous variation of the crossing angle and the speed of beam one, while the speed of beam two is fixed. As before, we assume $v_{2}=500 \mathrm{~m} \mathrm{~s}^{-1}$ and $\Delta v_{2}=50 \mathrm{~m} \mathrm{~s}^{-1}$. We calculate the values for $v_{1}$ and $\phi$ that result in an optimal energy resolution for the cases $\Delta_{\phi}=20,40,80 \mathrm{mrad}$ (hereafter referred to as case 1, 2 and 3 , respectively). In all cases and for all values for $v_{1}$ we assume $\Delta v_{1}=10 \mathrm{~m} \mathrm{~s}^{-1}$. The minimal value for $\Delta(E / \mu)$ has been determined by numerically evaluating eqn (6) on a sufficiently fine grid, subject to the condition of constant collision energy. In Fig. 5 the optimal values for $\Delta(E / \mu)$ are shown (red curves) as a function of the collision energy for all three cases. The values for $\phi$ (black curve) and $v_{1}$ (green curve) for a given $E / \mu$ are plotted with respect to the axis on the right.

To stay on the optimal curve, $\phi$ and $v_{1}$ have to be changed continuously. It is of practical interest to consider what happens if we move away from the optimal curve by either changing only $v_{1}$ or only $\phi$. In Fig. 6 such deviations are considered for case 1 . The solid lines correspond to a change of $v_{1}$ from 0 to $1000 \mathrm{~m} \mathrm{~s}^{-1}$ at fixed intersection angles (indicated on each curve). The two dashed lines correspond to fixed values for $v_{1}$ with $v_{1}=575$ or $773 \mathrm{~m} \mathrm{~s}^{-1}$ and variable $\phi$ with $\phi=0^{\circ}-90^{\circ}$.

All curves touch the optimal curve of case 1, as it should be. The energy range that can be scanned with a close to optimal resolution appears limited, both in the case where only $v_{1}$ is varied and in the case where only $\phi$ is varied. Note that by changing $v_{1}$ alone, the energy range with a satisfactory energy resolution becomes more and more narrow as $\phi$ decreases, finally vanishing at $\phi=0^{\circ}$.

Let us now consider an apparatus in which the beam intersection angle is fixed, but both beam velocities are variable. We assume $\phi=45^{\circ}$, as this beam intersection angle appears experimentally most feasible. Again, we assume the beam parameters pertaining to case 1, i.e., $\Delta_{v_{1}}=10 \mathrm{~m} \mathrm{~s}^{-1}$ for all values of $v_{1}, \Delta_{v_{2}}=0.10 \times v_{2}$, and $\Delta_{\phi}=20 \mathrm{mrad}$. In Fig. 7 the optimal values for $\Delta(E / \mu)$ are shown (red curve, labeled $\left(1^{\prime}\right)$ ), together with curve (1) that was shown in the preceding figures. On the left side of this figure, the corresponding values for $v_{1}$ and $v_{2}$ that are required to obtain the optimal value for the energy resolution are shown in green.

It is observed that by a proper variation of $v_{1}$ and $v_{2}$ at a fixed value of $\phi=45^{\circ}$ (curve $\left(1^{\prime}\right)$ ), energy resolutions are

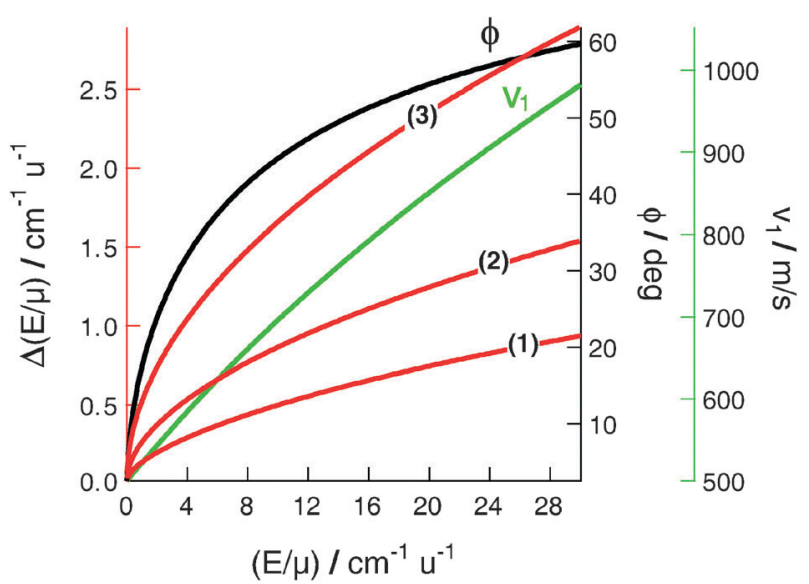

Fig. 5 The minimized values for $\Delta(E / \mu)$ (red curves with respect to the left axis) pertaining to the situation in which the target beam velocity $v_{2}$ is kept constant, and both $v_{1}$ and $\phi$ are allowed to vary to tune the collision energy. Beam parameters: $\Delta v_{1}=10 \mathrm{~m} \mathrm{~s}^{-1}, v_{2}=500 \mathrm{~m} \mathrm{~s}^{-1}$ and $\Delta_{v_{2}}=50 \mathrm{~m} \mathrm{~s}^{-1}$. The assumed angular spreads are $\Delta \phi=20,40$, $80 \mathrm{mrad}$ corresponding to curves 1,2 and 3 respectively. The values for $v_{1}$ (green curve) and for $\phi$ (black curve) that result in the optimal energy resolution are plotted with respect to the axis on the right. 


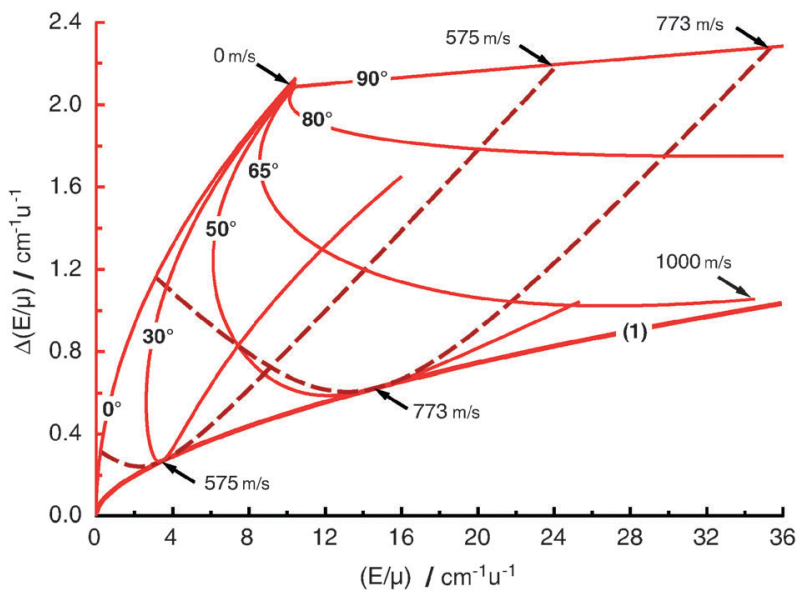

Fig. 6 The expected energy resolution if only one parameter is continuously varied in an experiment to tune the collision energy. Beam parameters: $\Delta v_{1}=10 \mathrm{~m} \mathrm{~s}^{-1}, v_{2}=500 \mathrm{~m} \mathrm{~s}^{-1}, \Delta_{v_{2}}=50 \mathrm{~m} \mathrm{~s}^{-1}$, and $\Delta \phi=20 \mathrm{mrad}$. Solid curves: $v_{1}$ is continuously varied between $0 \mathrm{~m} \mathrm{~s}^{-1}$ and $1000 \mathrm{~m} \mathrm{~s}^{-1}$ for fixed beam intersection angles of $0^{\circ}, 30^{\circ}$, $50^{\circ}, 65^{\circ}, 80^{\circ}$ and $90^{\circ}$. Dashed curves: $\phi$ is continuously varied between $0^{\circ}$ and $90^{\circ}$ for fixed primary beam speeds of $v_{1}=575 \mathrm{~m} \mathrm{~s}^{-1}$ and $v_{2}=$ $773 \mathrm{~m} \mathrm{~s}^{-1}$. The optimal energy resolution that is obtained if both $v_{1}$ and $\phi$ are tuned is shown as a comparison (curve (1); reproduced from Fig. 5).

obtained that are very similar to the optimal resolution that results from a variation of $v_{1}$ and $\phi$ at a fixed value of $v_{2}$ (curve (1)). At low collision energies curve $\left(1^{\prime}\right)$ is below curve (1). This is a consequence of the fixed value of $v_{2}=500 \mathrm{~m} \mathrm{~s}^{-1}$ that was assumed for curve (1); for curve $\left(1^{\prime}\right) v_{2}$ may assume values below $500 \mathrm{~m} \mathrm{~s}^{-1}$, improving the energy resolution.

Again, we may wonder what would happen if we move away from the optimal curve by either changing only $v_{1}$ or $v_{2}$. This situation is addressed on the right hand side of Fig. 7. The energy resolution is shown that is obtained if $v_{1}$ is varied between $0 \mathrm{~m} \mathrm{~s}^{-1}$ and $1200 \mathrm{~m} \mathrm{~s}^{-1}$, while fixed values for $v_{2}$ of $400,500,600$ or $700 \mathrm{~m} \mathrm{~s}^{-1}$ are assumed. Again, we assume $\Delta_{v_{1}}=10 \mathrm{~m} \mathrm{~s}^{-1}$ for all values of $v_{1}, \Delta_{v_{2}}=0.10 \times v_{2}$, and $\Delta_{\phi}=$ $20 \mathrm{mrad}$. It can be seen that as long as $v_{1}$ can be tuned continuously, it is sufficient to change $v_{2}$ in coarser steps in order to traverse the minimum curve $\left(1^{\prime}\right)$. This is of practical importance because the mean speed of a beam can easily be adjusted in larger steps by varying, for instance, the nozzle temperature.

It is also interesting to compare some of the above results to a direct numerical evaluation of $\Delta E$. To see whether the linear approximation is sufficiently accurate, we compare the calculations for the three cases shown in Fig. 5 to a direct numerical evaluation of $\Delta(E / \mu)$. For the latter we generate independent random numbers from Gaussian distributions for the angular and velocity spreads. All three cartesian velocity components of a beam are assumed to be independent, and the total angular spread is shared equally among both beams. The distributions for $\Delta(E / \mu)$ are then not strictly Gaussian in shape, but can be reasonably well approximated by a Gaussian in all cases. The resulting values for a few sample points are plotted as dots in Fig. 8. It is seen that the linear

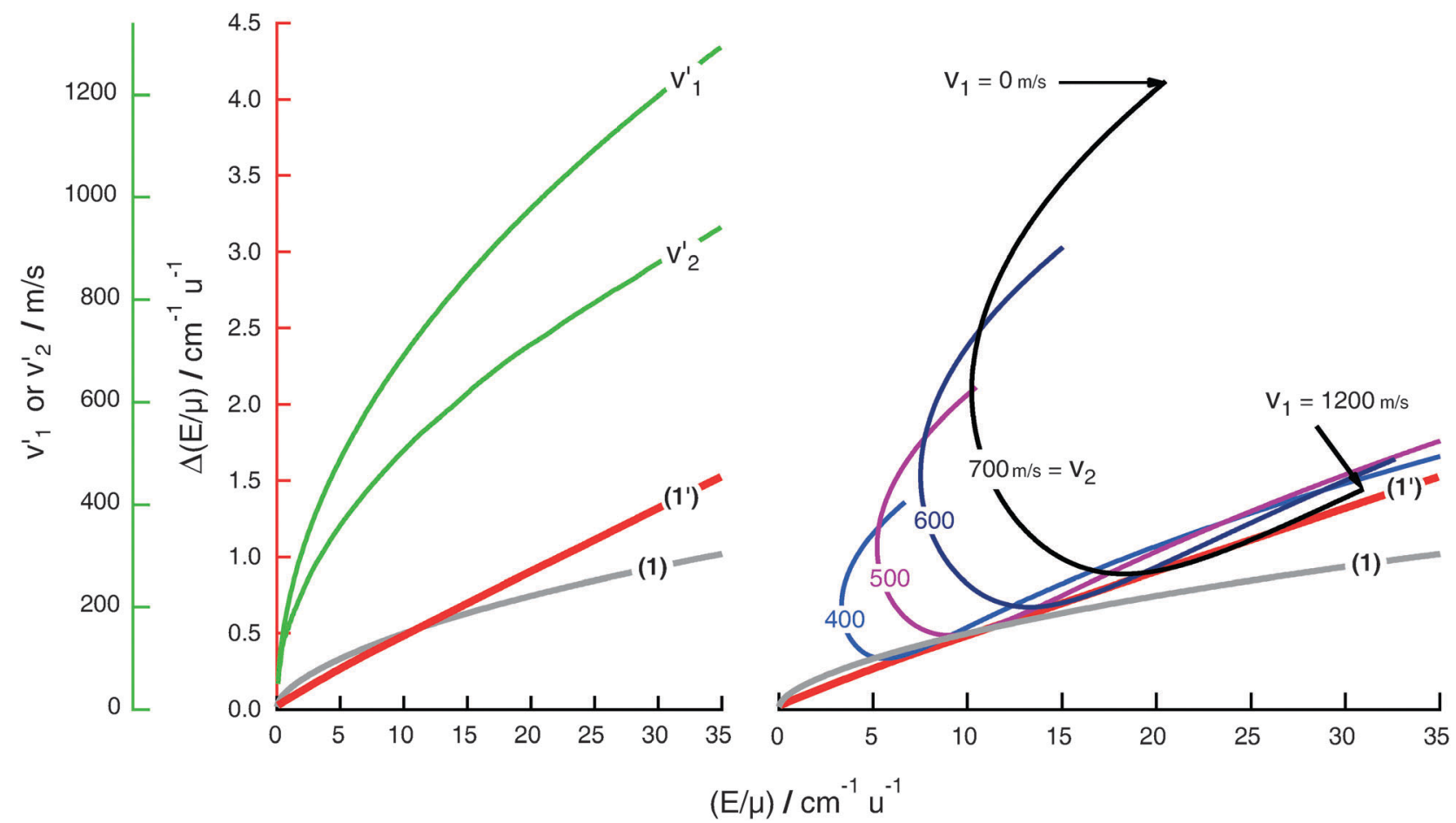

Fig. 7 The energy resolution for the situation in which both beam speeds $v_{1}$ and $v_{2}$ are varied for a fixed beam intersection angle $\phi=45^{\circ}$. Beam parameters: $\Delta v_{1}=10 \mathrm{~m} \mathrm{~s}^{-1}$ for all values of $v_{1}, \Delta v_{2}=0.10 \times v_{2}$, and $\Delta \phi=20 \mathrm{mrad}$. Left panel: optimized energy resolution $\left(\mathrm{curve}\left(1^{\prime}\right)\right)$. The values for $v_{1}$ and $v_{2}$ (green curves) that result in this energy resolution are shown with respect to the green axis on the left. Right panel: the energy resolution that is obtained if $v_{1}$ is continuously varied between 0 and $1200 \mathrm{~m} \mathrm{~s}^{-1}$ for fixed values of $v_{2}$ of $400,500,600$, and $700 \mathrm{~m} \mathrm{~s}$. For comparison, curve (1) of Fig. 5 is shown in both panels. 


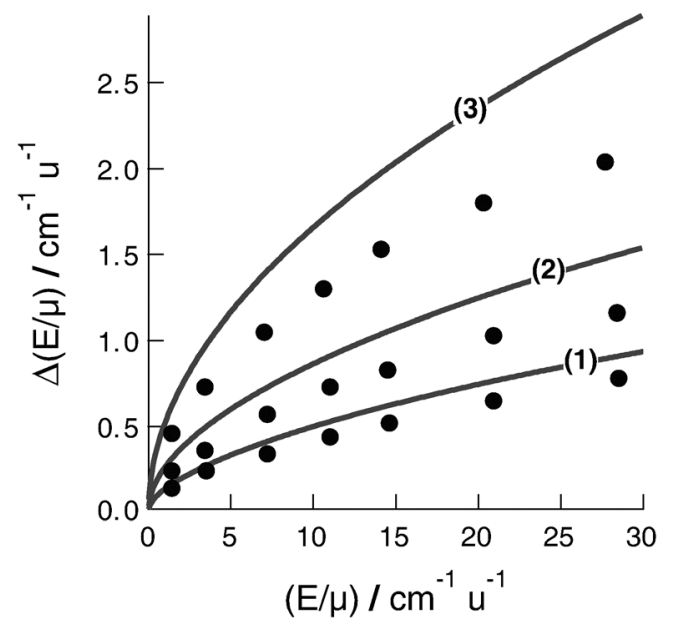

Fig. 8 The minimized values of $\Delta(E / \mu)$ pertaining to the three cases of Fig. 5. The values for $\Delta(E / \mu)$ are calculated using the model (curves labeled (1), (2) and (3); identical to the curves in Fig. 5) and direct numerical evaluation using Gaussian beam distributions (dots).

approximation indeed describes the considered cases well, but that it actually overestimates the numerically calculated spread $\Delta E / \mu$.

\section{Resonances in $\mathrm{OH}-\mathrm{He}$ collisions}

In this section, we illustrate the benefits of the method to improve the energy resolution, using the scattering of Starkdecelerated $\mathrm{OH}$ radicals with $\mathrm{He}$ atoms as an example. The scattering of $\mathrm{OH}$ with $\mathrm{He}$ is well-suited to be studied with high energy resolution, as this system is known to exhibit a pronounced resonant structure in the inelastic scattering cross sections. ${ }^{31}$ The potential well for the $\mathrm{OH}-\mathrm{He}$ van der Waals complex is shallow, and can only support a limited number of bound states. ${ }^{32,33}$ The well depth of $\sim 25 \mathrm{~cm}^{-1}$ is significantly smaller than the energy spacing between rotational levels of the $\mathrm{OH}$ radical, resulting in resonances in the inelastic cross sections that are grouped within rather narrow collision energy ranges. The well-defined range of collision energies at which scattering resonances can occur, in combination with the low number of resonances that can be expected within this range, yield interesting prospects to experimentally (partially) resolve these resonances.

Recently, we have studied the low-energy state-to-state rotationally inelastic scattering cross sections for this system by scattering a beam of Stark-decelerated $\mathrm{OH}$ radicals with a conventional beam of $\mathrm{He}$ atoms in a crossed molecular beam (90 degrees crossing angle) configuration. ${ }^{34}$ In this experiment, the velocity of the He atoms is $\sim 1000 \mathrm{~m} \mathrm{~s}^{-1}$, and the collision energy is tuned by varying the $\mathrm{OH}$ velocity using the Stark decelerator. In panel (a) of Fig. 9, the measured and the calculated relative cross sections for 3 inelastic scattering channels are shown. The $\mathrm{OH}$ molecules scatter out of the $X^{2} \Pi_{3 / 2}, J=3 / 2, f$ initial state into final states of the $X^{2} \Pi_{3 / 2}$ manifold with $J=3 / 2 e, 5 / 2 e, 5 / 2 f$. The labels $e$ and $f$ denote the lower and upper $\Lambda$-doublet component of the rotational levels, respectively. The cross sections that result from quantum close-coupled scattering calculations using high-quality ab initio $\mathrm{OH}-\mathrm{He}$ potential energy surfaces are shown in panel (a) as well.

Three groups of scattering resonances at collision energies around $126 \mathrm{~cm}^{-1}, 188 \mathrm{~cm}^{-1}$ and $202 \mathrm{~cm}^{-1}$ are clearly recognized in the calculated cross sections. These resonances can be associated with the potential wells of the $\mathrm{OH}-\mathrm{He}$ potential energy surfaces that adiabatically connect to the free He atom and the $X^{2} \Pi_{1 / 2}, J=1 / 2, X^{2} \Pi_{1 / 2}, J=3 / 2$, and $X^{2} \Pi_{3 /}$ ${ }_{2}, J=7 / 2$ rotational energy levels of the free $\mathrm{OH}$ radical, respectively. The collision energy resolution that was obtained in the experiment amounted to approximately $25 \mathrm{~cm}^{-1}$; the theoretically calculated cross sections, convoluted with the experimental energy resolution, are shown as black curves in panel (a) of Fig. 9. Clearly, the scattering resonances are smeared out due to the experimental energy spread, and no unequivocal resonant structure can be identified in the experimental cross sections.
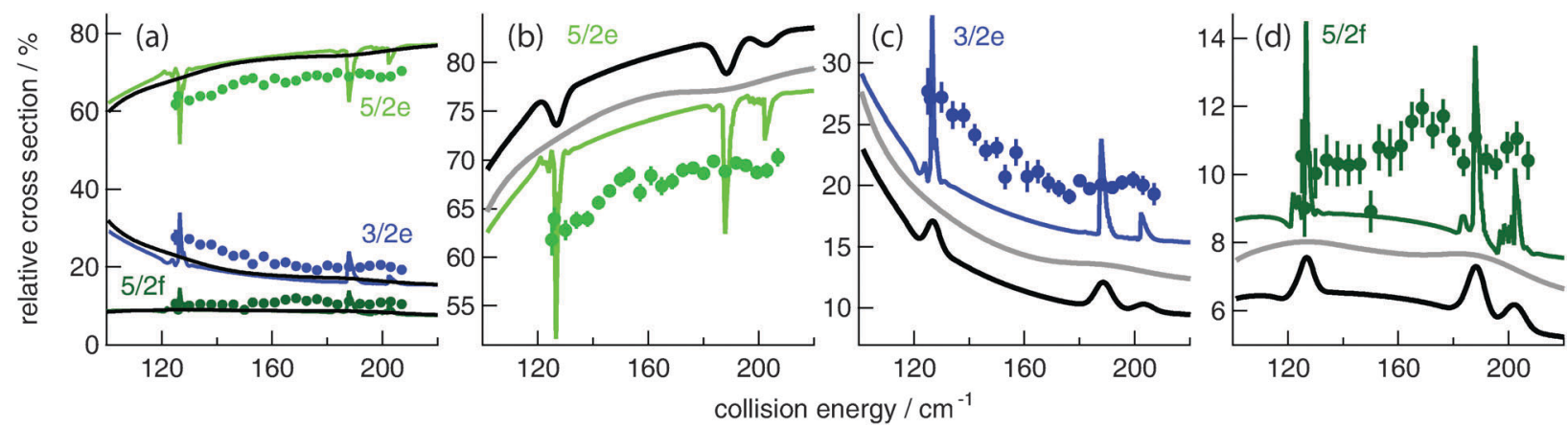

Fig. 9 (a) Measured and theoretically predicted relative cross sections for three inelastic scattering channels for the scattering of OH radicals $\left(X^{2} \Pi_{3 / 2}, J=3 / 2, f\right)$ with ${ }^{4} \mathrm{He}$ atoms (adapted from ref. 34). The theoretically predicted cross sections are shown as colored curves, while the black curves result if the calculated cross sections are convoluted with the experimental energy resolution. (b-d) Experimental cross sections together with the theoretically predicted relative cross sections, convoluted with the experimental energy resolution for two different experimental arrangements. The beam parameters pertain to case (1): $v_{\mathrm{He}}=1000 \mathrm{~m} \mathrm{~s}^{-1}, \Delta v_{\mathrm{He}}=100 \mathrm{~m} \mathrm{~s}^{-1}, \Delta v_{\mathrm{OH}}=10 \mathrm{~m} \mathrm{~s}^{-1}$ and $\Delta \phi=20 \mathrm{mrad}$. Gray curves: fixed beam crossing angle of $\phi=90^{\circ}$. Black curves: beam crossing angle $\phi$ and $\mathrm{OH}$ velocity $v_{\mathrm{OH}}$ are chosen to optimize the energy resolution at each collision energy. The vertical axes pertain to the theoretically predicted cross sections; the gray and black curves are vertically offset by a few percent for reasons of clarity. 
A straightforward strategy to improve the experimental energy resolution in this experiment would be to improve the angular and velocity spreads of both the $\mathrm{OH}$ radical and $\mathrm{He}$ atomic beams. A significant improvement of the beam parameters (and concomitant reduction in particle densities) is considered feasible regarding the excellent signal-to-noise ratio that has been attained in the experiment. In our crossed beam scattering experiments, beam parameters pertaining to case 1 of Section III, i.e., $\Delta v_{\mathrm{He}}=100 \mathrm{~m} \mathrm{~s}^{-1}\left(10 \%\right.$ of $\left.v_{\mathrm{He}}\right)$, $\Delta v_{\mathrm{OH}}=10 \mathrm{~m} \mathrm{~s}^{-1}$ and $\Delta \phi=20 \mathrm{mrad}$, are considered experimentally challenging but feasible.

In panels (b)-(d) of Fig. 9, the experimentally observed and calculated relative cross sections for the three inelastic scattering channels are shown again on an enlarged scale. The gray curves show the theoretically predicted cross sections convoluted with the experimental energy resolution that is expected if the experiment would be performed with the case (1) beam parameters. The vastly improved beam parameters only result in a slightly improved energy resolution. Like in panel (a), this energy resolution is not sufficient to resolve the scattering resonances, and one may wonder if the improved energy resolution that is obtained justifies the experimental effort of improving the beam parameters.

Things look considerably better if the methodology to improve the energy resolution as outlined in this paper is followed. In panels (b)-(d) of Fig. 9, the black curves show the cross sections that are expected experimentally if again case (1) beam parameters are used, but now the beam crossing angle $\phi$ and $v_{\mathrm{OH}}$ are chosen to optimize the resolution for every collision energy. Clearly, the improved energy resolution facilitates the experimental observation of the resonant structure in the scattering cross sections. At the resonance positions, the optimized parameters are: $E=126 \mathrm{~cm}^{-1}: \phi=44^{\circ}, v_{\mathrm{OH}}=$ $1388 \mathrm{~m} \mathrm{~s}^{-1} ; E=188 \mathrm{~cm}^{-1}: \phi=49.8^{\circ}, v_{\mathrm{OH}}=1544 \mathrm{~m} \mathrm{~s}^{-1} ; E=$ $202 \mathrm{~cm}^{-1}: \phi=50.8^{\circ}, v_{\mathrm{OH}}=1577 \mathrm{~m} \mathrm{~s}^{-1}$, resulting in an energy resolution $\Delta E$ of $5.2 \mathrm{~cm}^{-1}, 6.6 \mathrm{~cm}^{-1}$, and $6.9 \mathrm{~cm}^{-1}$, respectively. This resolution is sufficient to partially resolve the scattering resonances; a further improvement of the beam parameters will result in a further improvement of the energy resolution.

\section{Conclusion}

We have presented a simple yet effective method to optimize the collision energy resolution in crossed molecular beam scattering experiments. We show that for beam intersection angles smaller than $90^{\circ}$, kinematically favorable conditions can be found in which the beam with the largest velocity or angular spread contributes the least to the collision energy resolution. This allows for high collision energy resolutions, without the need for methods that reduce the velocity spread of the beam(s) and without greatly reducing the particle density in the beam(s). Via a systematic optimization of the beam velocities and beam intersection angle, we have analyzed the optimal value for the energy resolution that can be reached experimentally using a realistic set of beam parameters.

The method may offer particularly large dividends if one of the molecular beam pulses already has a narrow angular and velocity spread, as is the case for Stark-decelerated beams, for instance. Stark decelerators offer molecular packets with a tunable velocity, an angular spread of typically $1^{\circ}$, and a narrow velocity spread that is typically in the $5-20 \mathrm{~m} \mathrm{~s}^{-1}$ range. Using additional electric field elements with which the phase-space distribution of the molecules is manipulated, velocity spreads below $1 \mathrm{~m} \mathrm{~s}^{-1}$ can be obtained. ${ }^{30}$ Using a suitable beam intersection angle and velocity of the target beam, this narrow angular and velocity spread allows for exceptionally high collision energy resolutions. In particular for systems with a low reduced mass, absolute collision energy resolutions ranging from $0.5-5 \mathrm{~cm}^{-1}$ appear feasible. This may well be exploited to experimentally observe and study scattering resonances. Experiments of this kind are currently underway.

\section{Acknowledgements}

This work is supported by the ESF EuroQUAM programme, and is part of the CoPoMol (Collisions of Cold Polar Molecules) project.

\section{References}

1 R. Levineand R. Bernstein, Molecular reaction dynamics and chemical reactivity, Oxford University Press, New York, 1987.

2 X. Yang, Annu. Rev. Phys. Chem., 2007, 58, 433.

3 Atomic and molecular beam methods, ed. G. Scoles, Oxford University Press, New York, NY, USA, 1988 \& 1992, vol. 1 \& 2.

4 M. N. R. Ashfold, N. H. Nahler, A. J. Orr-Ewing, O. P. J. Vieuxmaire, R. L. Toomes, T. N. Kitsopoulos, I. A. Garcia, D. A. Chestakov, S.-M. Wu and D. H. Parker, Phys. Chem. Chem. Phys., 2006, 8, 26.

5 G. Hall, K. Liu, M. McAuliffe, C. Giese and W. Gentry, J. Chem. Phys., 1983, 78, 5260.

6 R. Macdonald and K. Liu, J. Chem. Phys., 1989, 91, 821.

7 D. M. Sonnenfroh, R. G. Macdonald and K. Liu, J. Chem. Phys., 1991, 94, 6508.

8 R. T. Skodje, D. Skouteris, D. E. Manolopoulos, S.-H. Lee, F. Dong and K. Liu, Phys. Rev. Lett., 2000, 85, 1206.

9 D. Skouteris, D. E. Manolopoulos, W. Bian, H. J. Werner, L. H. Lai and K. Liu, Science, 1999, 286, 1713.

10 S. Y. T. van de Meerakker, H. L. Bethlem and G. Meijer, Nat. Phys., 2008, 4, 595.

11 J. J. Gilijamse, S. Hoekstra, S. Y. T. van de Meerakker, G. C. Groenenboom and G. Meijer, Science, 2006, 313, 1617.

12 L. Scharfenberg, J. Kłos, P. J. Dagdigian, M. H. Alexander, G. Meijer and S. Y. T. van de Meerakker, Phys. Chem. Chem. Phys., 2010, 12, 10660.

13 M. Kirste, L. Scharfenberg, J. Kłos, F. Lique, M. H. Alexander, G. Meijer and S. Y. T. van de Meerakker, Phys. Rev. A: At., Mol., Opt. Phys., 2010, 82, 042717.

14 S. Y. T. van de Meerakker and G. Meijer, Faraday Discuss., 2009, $142,113$.

15 W. Erlewein, M. von Seggern and J. P. Toennies, Z. Phys., 1968, 211, 35.

16 D. W. Chandler, J. Chem. Phys., 2010, 132, 110901.

17 M. von Seggern and J. P. Toennies, Z. Phys., 1969, 218, 341.

18 R. N. Zare, Science, 2006, 311, 1383.

19 A. Schutte, D. Bassi, F. Tommasini and G. Scoles, Phys. Rev. Lett., 1975, 29, 979.

20 A. Schutte, D. Bassi, F. Tommasini and G. Scoles, J. Chem. Phys., $1975,62,600$

21 J. P. Toennies, W. Welz and G. Wolf, J. Chem. Phys., 1979, 71, 614.

22 M. H. Qiu, Z. F. Ren, L. Che, D. X. Dai, S. A. Harich, X. Y. Wang, X. M. Yang, C. X. Xu, D. Q. Xie, M. Gustafsson, R. T. Skodje, Z. G. Sun and D. H. Zhang, Science, 2006, 311, 1440.

23 W. Dong, C. Xiao, T. Wang, D. Dai, X. Yang and D. H. Zhang, Science, 2010, 327, 1501.

24 C. Berteloite, M. Lara, A. Bergeat, S. Le Picard, F. Dayou, K. H. Hickson, A. Canosa, C. Naulin, J.-M. Launay, I. R. Sims and M. Costes, Phys. Rev. Lett., 2010, 105, 203201. 
25 H. L. Bethlem, F. M. H. Crompvoets, R. T. Jongma, S. Y. T. van de Meerakker and G. Meijer, Phys. Rev. A: At., Mol., Opt. Phys., 2002, 65(5), 053416.

26 H. Pauly and J. P. Toennies, Methods of Experimental Physics, Academic Press, New York and London, 1968, vol. 7a, p. 296.

27 R. Feltgen, Weitere Messungen der Geschwindigkeitsabhängigkeit des totalen Streuquerschnittes an Systemen mit kleiner reduzierter Masse, 1970, PhD thesis, available via the library of the Bonn University.

28 M. S. Elioff, J. J. Valentini and D. W. Chandler, Science, 2003, 302, 1940.

29 M. S. Elioff, J. J. Valentini and D. W. Chandler, Eur. Phys. J. D, 2004, 31, 385.
30 F. M. H. Crompvoets, R. T. Jongma, H. L. Bethlem, A. J. A. van Roij and G. Meijer, Phys. Rev. Lett., 2002, 89(9), 093004.

31 J. Kłos, F. Lique and M. H. Alexander, Chem. Phys. Lett., 2007, 445, 12. 32 J. Han and M. C. Heaven, J. Chem. Phys., 2005, 123, 064307.

33 H.-S. Lee, A. B. McCoy, R. R. Toczyłowski and S. M. Cybulski, J. Chem. Phys., 2000, 113, 5736.

34 M. Kirste, L. Scharfenberg, J. Kłos, F. Lique, M. H. Alexander, G. Meijer and S. Y. T. van de Meerakker, Phys. Rev. A: At., Mol., Opt. Phys., 2010, 82, 042717.

35 Derivation available upon request.

36 Defined as usual by $\sigma^{2}=\frac{1}{n} \sum_{i}\left(\bar{x}-x_{i}\right)^{2}$, with the average value of $x$ denoted by $\bar{x}$, and the summation over the total number of particles $n$. 